\title{
Cryo-STEM-EDX for Reliable Characterization of Sulfur Distribution and the Rational Design of Sulfur Hosts for Li-S Batteries
}

\author{
Yao Yang ${ }^{1}$, Barnaby Levin ${ }^{2}, \mathrm{Na}_{\text {Zhang }}{ }^{1}$, Héctor Abruña ${ }^{1}$ and David Muller ${ }^{1}$ \\ ${ }^{1}$ Cornell University, Ithaca, New York, United States, ${ }^{2}$ Arizona State University, Tempe, Arizona, United \\ States
}

Lithium-sulfur (Li-S) batteries have emerged as one of the most promising candidates for next-generation renewable energy storage systems. ${ }^{\mathbf{1 - 3}}$ Sulfur can provide a high theoretical specific capacity (1675 $\mathrm{mAh} / \mathrm{g}$ ), ten times higher than that of commercial Li-ion batteries (LIBs). Sulfur is an earth-abundant element with very low cost $(<\$ 100 /$ ton $)$. Despite these advantages, a number of challenges must be overcome in order to develop commercially viable Li-S batteries, including the insulating nature of sulfur, and the highly soluble nature of some lithium polysulfides (LPSs) formed during discharge. LPSs can also easily "shuttle" between cathode and anode, leading to self-discharge, loss of active material and rapid capacity fade. To tackle these challenges, various hosts have been developed to immoblize the sulfur/LPSs, such as, porous carbons, metal oxides/sufides, and metal organic frameworks (MOFs). ${ }^{3-4}$

Evaluating the effectiveness of the host requires accurate characterization of the microstructures of sulfurhost composites. Scanning/transmission electron microscopy (S/TEM) at room temperature is routinely used to study sulfur-host composites. ${ }^{\mathbf{5 - 9}}$ However, sulfur is a beam-sensitive soft material and prone to sublimation under the typical vacuum conditions $\left(10^{-7} \sim 10^{-8}\right.$ torr) of SEM or TEM (Fig. 1a). ${ }^{\mathbf{1 0 , 1 1}}$ Recent studies have begun to make the Li-S battery community aware of the fact that sulfur sublimation, in S/TEM, can cause loss of sulfur from a sulfur cathode, as well as a redistribution of sulfur within the electrode composite, due to the capture of sublimated sulfur gas by porous materials. ${ }^{\mathbf{1 2}-14}$ Characterization of sulfur cathodes by room temperature S/TEM may therefore give misleading results, and alternative methods are necessary to advance our design of sulfur-host cathodes.

We have employed cryo-S/TEM equipped with energy dispersive X-ray spectroscopy (EDX) to reliably characterize the intrinsic morphology and structure of sulfur, as well as its distribution in a variety of sulfur hosts without sulfur sublimation artifacts. ${ }^{\mathbf{1 5 - 1 7}}$ Compared to traditional TEM, cryo-TEM has been shown to effectively suppress sulfur sublimation by keeping the sample near liquid N2 temperatures (-183 $\left.{ }^{\mathrm{O}} \mathrm{C}\right) .{ }^{13} \mathrm{EDX}$ in STEM mode enables elemental mapping of up to $\mu \mathrm{m}$-thick sulfur with nm-scale resolution using the sulfur K-edge $(2.3 \mathrm{keV})$ and quantitative elemental analysis in sulfur-host composites. In contrast, sulfur particles are often too thick for electron energy loss spectroscopy (EELS).

Sulfur has been traditionally mixed with conducting hosts through a melt-infusion method, in which sulfur melts at $155^{\circ} \mathrm{C}$ and slowly infuses into hosts. ${ }^{\mathbf{5 - 9}}$ Although porous carbon materials were first reported as sulfur hosts, ${ }^{1}$ the capacity decay using such hosts is still severe after moderate cycling, likely due to the poor interaction of non-polar carbons with the polar sulfur/LiPSs. We have shown that metal sulfides and oxides, with strong chemical interactions with sulfur/LiPSs, can significantly enhance cycling performance. ${ }^{15-17}$ As shown in Figs. 1b-c, the cryo-STEM images and EDX maps exhibit well-faceted $\mu \mathrm{m}$-sized sulfur particles overlapping a layered metal sulfide $\left(\mathrm{TiS}_{2}\right)$ host. ${ }^{15}$ Similarly, when porous metal oxides $\left(\mathrm{Fe}_{3} \mathrm{O}_{4}\right)$ were used as sulfur hosts, $\mu \mathrm{m}$-sized sulfur particles were embedded in a conductive network of $\mathrm{Fe}_{3} \mathrm{O}_{4}$ hosts (Fig. 1d-f). Despite the $\mathrm{Fe}_{3} \mathrm{O}_{4}$ pores being filled with sulfur, the majority of sulfur 
remained outside. ${ }^{\mathbf{1 6}}$ Those two examples, together with our previous finding of a similar morphology of sulfur in porous carbon hosts, ${ }^{\mathbf{1 4}}$ suggest that a universal morphology, of well-faceted $\mu \mathrm{m}$-sized sulfur particles, is formed with porous hosts via the melt-infusion method. This is in contrast to previous reports on similar materials, using room-temperature TEM, that observed nm-sized sulfur particles, and/or complete diffusion of sulfur into the void space. ${ }^{\mathbf{5 - 9}}$ Our work presented here calls for caution when evaluating TEM analysis of sulfur-host composites without cryogenic conditions.

Here, cryo-STEM-EDX was employed to further guide the design of more effective hosts (Fig. 2). ${ }^{\mathbf{1 7}} \mathrm{We}$ developed a novel sulfur confinement approach by in situ encapsulating sulfur with a MOF-derived $\mathrm{CoS}_{2}$ in a conductive carbon matrix $\left(\mathrm{S} / \mathrm{Z}-\mathrm{CoS}_{2}\right)$, which in turn was derived from a non-conductive sulfur/zeolitic imidazolate framework-67 (S/ZIF-67) composite via heat treatment. Cryo-STEM-EDX of S/ZIF-67 and S/Z-CoS 2 exhibited a highly homogenous distribution of $S$ and Co in ZIF particles (Figs. 2a-f). EDX mapping and quantitative analysis of S/ZIF-67 and S/Z-CoS 2 suggested that sulfur resided inside the ZIFderived cages, rather than externally (Figs. 2c,f). A control group of hollow ZIF-67 was prepared in order to compare our integrated hosts with traditional sulfur melt-infusion strategies, which are widely used for hollow/porous hosts (Fig. 2h). Hollow ZIF-67 was infiltrated with sulfur to form sulfur/hollow ZIFderived $\mathrm{CoS} 2$ composites (S/H-CoS2, Fig. 2i). Cryo-STEM-EDX showed that, with a sulfur mass fraction of $70 \%$, a significant amount of sulfur in $\mathrm{S} / \mathrm{H}-\mathrm{CoS}_{2}$ stays outside of the host after filling all the void space in $\mathrm{H}-\mathrm{CoS}_{2}$ (Fig. 2j). S/Z-CoS composites exhibited highly improved battery durability after 1000 cycles at a current rate of $1 \mathrm{C}$, relative to both $\mathrm{S} / \mathrm{ZIF}-67$ and $\mathrm{S} / \mathrm{H}-\mathrm{CoS}_{2}$ (Fig. 2k). This improved performance was ascribed to not only efficient physical confinement of sulfur inside the host, but also to strong chemical interactions between $\mathrm{CoS}_{2}$ and sulfur/LPSs as well as enhanced electronic conductivity.

In summary, cryo-S/TEM-EDX enables the reliable characterization of well-defined crystalline $\mu \mathrm{m}$-sized sulfur in various hosts through a traditional melt-infusion method. Cryo-STEM serves as a powerful methodology to guide the design of a novel MOF-based $\mathrm{CoS}_{2}$ host with a complete encapsulation of sulfur and strong chemical interactions, benefiting future development of high-capacity and long-life Li-S batteries. $^{18}$ 

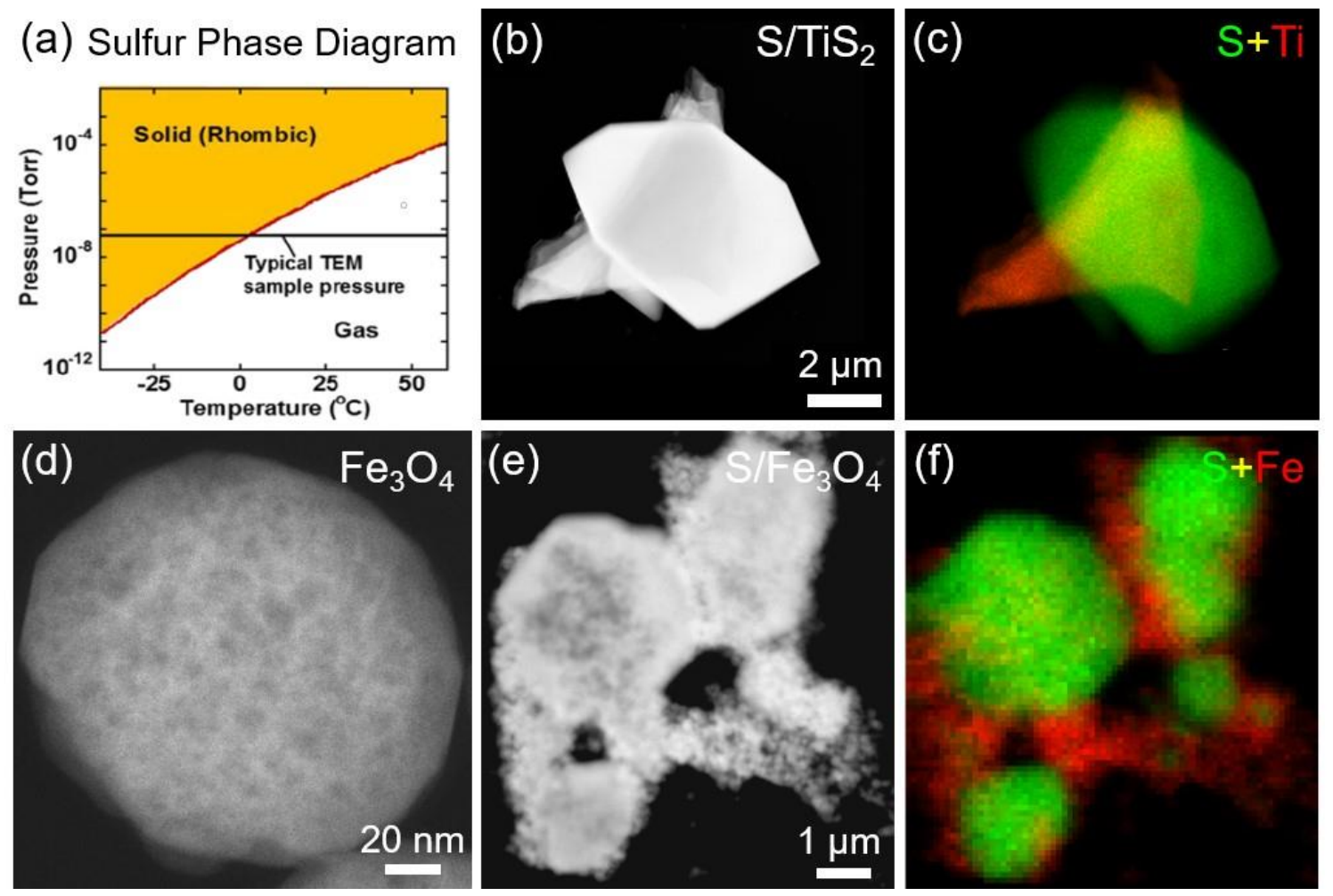

Figure 1. Cryo-STEM-EDX enables the reliable characterization of sulfur with various host materials (a) Low-pressure phase diagram of sulfur, indicating that sulfur is in the gas phase at room temperature under the high vacuum conditions of TEM. (b-c) Cryo-STEM images and EDX elemental maps of S/TiS2 composites with well-faceted $\mu \mathrm{m}$-sized sulfur on top of layered TiS2.15 (d) ADF-STEM image of porous Fe3O4 nanoparticles ( 100 nm). (e-f) Cryo-STEM and EDX of S/Fe3O4 composites16 with similar morphology of sulfur as TiS2 and porous carbon hosts revealed by our previous cryo-STEM studies, 14,15 which is in contrast to other literature reports of nm-sized sulfur observed in conventional TEM at room temperature. 


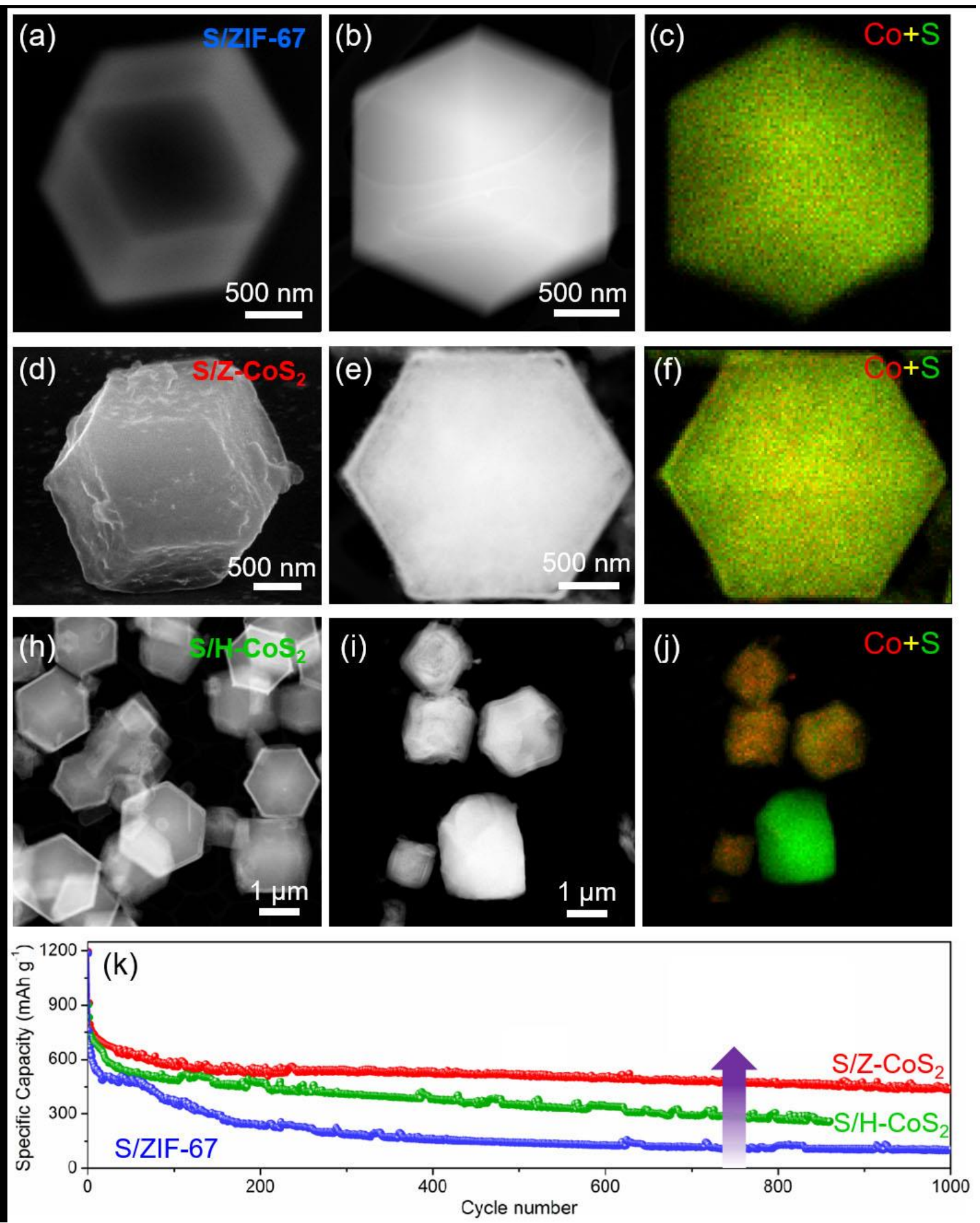

Figure 2. Cryo-STEM-EDX guides the design of novel MOF-based sulfur hosts.17 (a-c) and (d-f) CryoSEM, STEM images and EDX maps of insulating S/ZIF-67 and conductive S/Z-CoS2 composites, which represents $\mathrm{CoS} 2$ in a carbon matrix derived from S/ZIF-67 via heat treatment. (h-j) Cryo-STEM images of hollow CoS2 and S/hollow CoS2 composites (S/H-CoS2) and EDX maps of S/H-CoS2, which served as a control group to represent hollow/porous hosts used to contain sulfur through traditional sulfur infiltration method. (k) Long-term battery cycling performance of S/Z-CoS2, S/H-CoS2 and S/ZIF-67 for 1000 cycles at a current rate of $1 \mathrm{C}$, with the purple arrow highlighting the superior performance of S/Z- 
CoS2, owing to a complete sulfur encapsulation inside MOF-based cages, strong chemical interaction between $\mathrm{CoS} 2$ and sulfur/polysulfides and enhanced electronic conductivity.

\section{References}

1. Ji, K. T. Lee, L. F. Nazar, Nat. Mater. 8 (2009), 500.

2. Manthiram, Y. Fu, S.H. Chung, C. Zu, Y.S. Su, Chem. Rev. 114 (2014), 11751.

3. Li, H. B. Wu, X. W. Lou, Energy Environ. Sci. 9 (2016), 3061.

4. Evers, L.F. Nazar, Acc. Chem. Res. 46 (2013), 1135.

5. Liu, J. Q. Huang, Q. Zhang, L. Mai, Adv. Mater. 29 (2017), 1601759.

6. W. Seh, W.Li, J. J.Cha, G. Zheng, Y. Yang, M.T. McDowell, P.C. Hsu, Y. Cui, Nat. Commun. 4 (2013), 1331.

7. Fan, W. Liu, Z. Weng, Y. Sun, H. Wang, J. Am. Chem. Soc. 137 (2015), 12946.

8. Li, J. Zhang, X.W. Lou, Angew. Chem. Int. Ed. 54 (2015), 12886.

9. Xin, L. Gu, N. H. Zhao, Y. X. Yin, L. J. Zhou, L.-J. Wan, J. Am. Chem. Soc. 134 (2012), 18510.

10. B. Nash, Icarus, 72 (1987), 1.

11. G. M. Ferreira, L.Q. Lobo, J. Chem. Thermodyn. 43 (2011), 95.

12. Raiß, K. Peppler, J. Janek, P. Adelhelm, Carbon 79 (2014), 245.

13. D. A. Levin, M. J. Zachman, J. G. Werner, R. Sahore, K. X. Nguyen, Y. Han, B. Xie, L. Ma, L. A. Archer, E. P. Giannelis, U. Wiesner, L. F. Kourkoutis, D. A. Muller, Microsc. Microanal. 23 (2017), 155. 14. Sahore, B.D.A. Levin, M. Pan, D.A. Muller, F.J. DiSalvo, E.P. Giannelis, Adv. Energy Mater. 6 (2016), 1600134.

15. -C. Liu, Y. Yang, J. Wu, M. Liu, S. P. Zhou, B. D. A. Levin, X.-D. Zhou, H. Cong, D. A. Muller, P. M. Ajayan, H. D. Abruña, F.-S. Ke, ACS Energy Lett. 3 (2018), 1325.

16. Zhang, B. D. A. Levin, Y. Yang, D. A. Muller, H. D. Abruña, J. Electrochem. Soc. 165 (2018), 165, A1656-A1661.

17. Zhang, Y. Yang, X. Feng, S.-H. Yu, J. Seok, D. A. Muller, H. D. Abruña. J. Mater. Chem. A 7 (2019), 21128.

18. This work was supported as part of the Energy Materials Center at Cornell, funded by the U.S. Department of Energy, under Award No. DE-SC0001086. This work made use of TEM facilities of the Cornell Center for Materials Research, supported by NSF MRSEC program (DMR-1719875). 